\title{
Pupil Size and Reactivity in Pediatric Patients with Sickle Cell Disease
}

\author{
Joana Mack ${ }^{1}$, Sara Frankowski ${ }^{2}$, Bethany Verkamp ${ }^{2}$, Shelley Crary ${ }^{2}$, Beverly Spray ${ }^{3}$, Lee \\ Crawley $^{1}$, Suzanne Saccente ${ }^{2}$, and Kathleen Neville ${ }^{4}$ \\ ${ }^{1}$ Arkansas Children's Hospital \\ ${ }^{2}$ University of Arkansas for Medical Sciences \\ ${ }^{3}$ Arkansas Children's Research Institute \\ ${ }^{4}$ University of Arkansas System
}

September 24, 2020

\begin{abstract}
Background/Objectives: Pupil size and reactivity have been studied in adult and pediatric populations to objectively measure pain which showed that pupillometry is a promising biomarker for pain measurement. Vaso-occlusive crisis, is a common complication of sickle cell disease (SCD) and better assessment tools are needed. The objective of this study is to establish normative values for pupil size and reactivity in pediatric patients with SCD with the hope that pupillometry can be used as a tool to objectively measure pain and response to treatment. Design/Methods: Institutional review board (IRB) approved prospective study enrolled 82 patients with SCD. Readings were performed using a NeurOptics PLR-2000 pupillometer. The following measures were collected: resting pupil size, $\%$ change in pupil size, pupil constriction velocity, and pupil dilation velocity. Results: Forty-four males and 38 females, all black, were studied. Median age was 11 years (range:2-21). The mean minimum and maximum pupil sizes were $3.52 \mathrm{~mm}$ and $5.08 \mathrm{~mm}$, respectively. The mean dilation velocity and maximum constriction velocity were $1.03 \mathrm{~mm} / \mathrm{s}$ and $-4.48 \mathrm{~mm} / \mathrm{s}$, respectively. When comparing our participants with white participants in a previously published pediatric study, there was a significant difference in maximum constriction velocity, maximum pupil size, and minimum pupil size.1 There was no significant difference when comparing means for these parameters. Conclusion: There was no significant difference in pupil size and reactivity between patients with SCD and black patients without the disease. Further investigation of pupillometry within the black population during vaso-occlusive crisis and in the "well state" is warranted in pediatric patients with SCD.
\end{abstract}

\section{Introduction}

Pupil size and reactivity have been studied in the adult and pediatric populations in an attempt to objectively quantify pain. Previously conducted studies have demonstrated pupillometry as a promising biomarker for objective pain measurement. ${ }^{1-3}$ A 2012 study demonstrated the significant correlation between patient verbally reported pain and pupillary dynamics in order to guide narcotic administration. ${ }^{2}$ Another study showed that pupillometry is a more sensitive test for pain assessment and response to medication than other hemodynamic markers, including vital signs. ${ }^{3}$ Factors including patient age, sex, and ethnicity have been previously studied in order to better understand the potential variables affecting pupil dynamics and establish a set of normal values. Regarding pupillary dynamics in younger patients, Brown et al. evaluated the pediatric population and demonstrated a slight change in pupil dynamics across pediatric age groups as well as differences across various ethnicities. ${ }^{1}$

Pupillometry is conducted using a hand-held device that measures a variety of pupil dynamics following exposure to an infrared light stimulus. The parameters of pupil constriction and dilation velocity as well as 
minimum and maximum pupil sizes are the most significant categories used for comparison of patient populations. The testing is noninvasive, quick, and carries little to no risk for the patient, making pupillometry an ideal tool for pain measurement.

The clinical application of pupillometry is particularly useful in the young pediatric population as it is more difficult to understand subjective patient-reported pain, and an objective pain evaluation measurement is needed. For example, in the pediatric sickle cell disease population, vaso-occlusive crisis is a common complication that presents with acute pain. Vaso-occlusive pain crises are often over or undertreated due to fear of medication side-effects, possible addiction, and variable tolerance. ${ }^{4}$ Quantifying acute pain and response to treatment has been difficult due to dependence on subjective measures. In this study, we aim to measure pupillometry measurements in pediatric patients with sickle cell disease as a first step to establish normative data for future utilization in cases of acute pediatric vaso-occlusive pain crisis. ${ }^{1}$

\section{Methods}

The University of Arkansas for Medical Sciences (UAMS) IRB approved this prospective, single-center study. Male and female patients ages 2-21 years were approached at Arkansas Children's Hospital Sickle Cell clinic during their outpatient visits. Patients with sickle cell disease (SS, SC, $\mathrm{SB}^{\mathrm{O}}$-thalassemia, $\mathrm{SB}^{+}$-thalassemia) were considered for the study and screened for enrollment. Patients were eligible if they were afebrile for 48 hours (fever defined as temperature $>101^{\circ} \mathrm{F}$ ), had vital signs within normal limits, had not received any narcotics in the last 24 hours, and were not currently experiencing any pain based on a patient-reported, age-appropriate pain scale. Additionally, patients were excluded if they had a prior history of stroke or neurological deficits.

After consent was obtained, each child was tested using the NeurOptics PLR-2000 Pupillometer. The handheld device includes a rubber cup that is placed over the child's eye in order to block any outside light sources. The device then locates the pupil and measures the resting (maximum) pupil diameter (mm). Next, an infrared light briefly flashes and the pupillometer measures the average and maximum pupil constriction velocity $(\mathrm{mm} / \mathrm{s})$, minimum pupil diameter $(\mathrm{mm}), \%$ change in pupil size in response to light, and the pupil dilation velocity after exposure to infrared light stimulus (mm/s) (Fig. 1). The testing takes $<2$ minutes, is noninvasive, and is associated with little to no risk for the patient. Patient data was excluded if a reading could not be obtained after 4 measurement attempts.

After data collection, the measurements were divided across different age groups as well as male vs. female study participants for analysis. Maximum and minimum pupil sizes were reported as means with standard deviations across six age groups (range 2-21 years old). Maximum and minimum pupil sizes, maximum and average constriction velocity, and dilation velocity were reported as means with standard deviation and $\mathrm{p}$ values as overall, male, and female results.

Data were evaluated for assumptions of normality and equal variances using the Shapiro-Wilk W test and Levene's tests, respectively. To determine if pupillometry measures differed between gender, independent sample t-tests were conducted. Minimum and maximum pupil sizes were presented as means and standard deviations. The GPLOT procedure, incorporating linear regression, was utilized to create scatter plots and regression lines for pupil size and velocity by patient age. All data were analyzed using SAS software version 9.4 (SAS Institute, Cary, NC, USA).

\section{Results}

A total of 82 participants, 44 males and 38 females, enrolled in the study. All study participants were black with a median age of 11 years old (range 2-21). In order to analyze the data for potential differences, measurements were extrapolated and compared between age groups and biological sex. The summary of all measured pupil dynamics and corresponding mean values with reported p-values can be seen in Table 1. The overall mean minimum and maximum pupil sizes were $3.52 \mathrm{~mm}$ and $5.08 \mathrm{~mm}$, respectively. There was no statistically significant correlation in minimum and maximum pupil size with age (Fig. 2). The mean dilation velocity and maximum constriction velocity were $1.03 \mathrm{~mm} / \mathrm{s}$ and $-4.48 \mathrm{~mm} / \mathrm{s}$, respectively. No 
statistical significant correlation was found between dilation velocity, maximum constriction velocity and age (Fig. 2). There was no statistically significant difference between males and females for all pupil dynamics. A breakdown of participant ages and corresponding mean maximum and minimum pupil sizes can be seen in Table 2.

The Brown et al. study reported a mean maximum constriction velocity of $-4.42 \mathrm{~mm} / \mathrm{s}$, mean maximum pupil size of $4.97 \mathrm{~mm}$, and mean minimum pupil size of $3.4 \mathrm{~mm}$ for their healthy black participants revealing no significant difference compared to our means $(\mathrm{t}=0.46 ; \mathrm{p}=0.645),(\mathrm{t}=1.25, \mathrm{p}=0.216),(\mathrm{t}=1.84$, $\mathrm{p}=0.069)$, respectively. However, comparing our participants with their white participants, there was a significant difference in maximum constriction velocity $(\mathrm{t}=3.45 ; \mathrm{p}=0.009)$, maximum pupil size $(\mathrm{t}=$ $-5.57 \mathrm{~mm}, \mathrm{p}<0.00001)$, and minimum pupil size $(\mathrm{t}=-3.24, \mathrm{p}=0.002)$.

\section{Discussion}

The use of pupillometry as an objective method to measure pain and analgesic response has proven to be a useful modality in a variety of populations; however, establishing normative values for select populations is still evolving as variables such as age, ethnicity, or certain comorbidities have shown variability in comparative measurements. ${ }^{1-3}$ This study suggests that when using pupillometry as a clinical tool in pediatric patients with sickle cell disease, it is reasonable to use the normative values established in previous studies of healthy participants with similar age and race.

Previous studies have shown significant differences in measurements when comparing age groups of healthy participants. With age, pupil size and dilation velocity increase slightly while maximum constriction velocity decreases. These changes with age are fairly correlated with changes in eye size but may also be influenced by developmental changes of the pupillary reflex. ${ }^{1,5}$ In the study by Brown et al., there were also significant differences in pupil size and maximum constriction velocity when comparing healthy black to their respective white cohorts. Our results also reflect a variation amongst races as our data showed no significant change when comparing children with sickle cell disease to healthy black children of a similar age, but did show a significant difference amongst our participants in contrast to their white cohorts in the aforementioned study.

When comparing various comorbidities to baseline values obtained from healthy participants of similar age and race, some disease processes have reflected significant changes, making it difficult to use previously established normative values for those specific populations. For example, children with ADHD have been shown to have higher pupillary velocity values in correlation with changes in thickness of the retinal nerve fiber layer and anxiety has been correlated with increased pupil sizes. ${ }^{6,7}$ Specific disease processes affecting the retina, such as diabetic retinopathy, have expectedly shown progressive changes in pupillary dynamics with increasing disease severity. ${ }^{8}$ Given the lack of a significant difference in our data when contrasted with Brown et al.'s black participants, our study supports the use of previously established normative values when using pupillometry in the assessment of children with sickle cell disease, as it does not appear to influence pupil dynamics.

Our data was obtained during outpatient clinic visits when participants were presumed to be at their baseline state of health and without significant pain or recent opioid use. This was done to first ensure that sickle cell disease did not lead to baseline changes in pupil dynamics prior to future investigations regarding changes during an acute vaso-occlusive pain crisis and its associated management with narcotics.

Vaso-occlusive crisis is unique to the sickle cell and is a major contributor to disease morbidity that is often difficult to treat appropriately. These crises are characterized by nociceptive pain and associated inflammation which can lead to severe sequelae such as multiorgan failure and even death. ${ }^{9}$ Opioids are a cornerstone of treatment during an acute pain crisis, but the assessment of pain and treatment efficacy is challenging as current measurements rely on subjective measurements that are not all encompassing and difficult for younger or non-verbal children to utilize. Due to concern of inadequate pain treatment and concern for adverse effects of opioids, pain is often under or over treated, hence the need for a reliable, objective method of pain measurement in this population. ${ }^{9}$ 
In a recent study by Connelly et al. self-reported pain levels in healthy children undergoing surgical procedures showed significant associations with several measures of pupillary response. Amongst these measures, maximum pupillary constriction velocity, a dynamic measurement of pupil response to a light stimulus, showed the most promise as a surrogate marker of pain. Their data showed an increase of 2 or more on the validated visual analog scale (VAS) was associated with a change of [?] $0.22 \mathrm{~mm} / \mathrm{sec}$ in maximum constriction velocity. As expected, an association between drug dose (in morphine equivalents) and pupil size was observed; however, when controlled for opioid effect, an independent effect of pain on pupil response persisted. ${ }^{10}$ Despite the known effect of opioids on pupil size, changes in dynamic pupil parameters have continued to be observed following titration of opioid dose to a level that elicits respiratory depression, and intraoperatively, changes in pupillary reflex dilation in children have occurred prior to significant changes in heart rate or blood pressure, suggesting that pupillometry could be a valid tool in not just initial assessment of pain level, but also when evaluating analgesic response. ${ }^{11,12}$

Patients with a history of stroke or other neurological deficits were not included therefore normative values found in this study cannot be extended to that particular population. Another limitation to this study was that every patient was black. As the population become more interracial, there needs to be further studies looking at their specific normative pupillometry values.

In conclusion, black pediatric patients with sickle cell disease have no significant difference in pupil size and reactivity compared to black pediatric patients without sickle cell disease but have a significant difference compared to white participants in the Brown et al study. Pupillometry studies are needed in pediatric patients with sickle cell disease in an acute vaso-occlusive crisis and are currently in progress at our institution.

\section{Conflict of Interest}

The authors declare that there is no conflict of interest.

\section{Acknowledgements}

Thank you to patients who participated in this study.

\section{Data Availability Statement}

The data that support the findings of this study are available on request from the corresponding author. The data are not publicly available due to privacy or ethical restrictions.

\section{References}

1. Brown JT, Connelly M, Nickols C, Neville KA. Developmental Changes of Normal Pupil Size and Reactivity in Children. J Pediatr Ophthalmol Strabismus . 2015;52(3):147-151. doi:10.3928/01913913-20150317-11

2. Aissou M, Snauwaert A, Dupuis C, Atchabahian A, Aubrun F, Beaussier M. Objective Assessment of the Immediate Postoperative Analgesia Using Pupillary Reflex Measurement: A Prospective and Observational Study.Anesthesiology . 2012;116(5):1006-1012. doi:10.1097/ALN.0b013e318251d1fb

3. Barvais L, Engelman E, Eba JM, Coussaert E, Cantraine F, Kenny GN. Effect site concentrations of remifentanil and pupil response to noxious stimulation. British Journal of Anaesthesia . 2003;91(3):347-352. doi:10.1093/bja/aeg178

4. Wang, CJ, Kavanagh, PL, Little, AA. Quality-of-care indicators for children with sickle cell disease. Pediatrics 2011; 128: 484-493. doi: 10.1542/peds.2010-1791

5. Shah SS, Ranaivo HR, Mets-Halgrimson RB, Rychlik K, Kurup SP. Establishing a normative database for quantitative pupillometry in the pediatric population. BMC Ophthalmol . 2020;20(1):121. doi:10.1186/s12886-020-01389-x

6. Aslan MG, Uzun F, Fındık H, Kaçar M, Okutucu M, Hocaoğlu Ç. Pupillometry measurement and its relationship to retinal structural changes in children with attention deficit hyperactivity disorder.Graefes Arch Clin Exp Ophthalmol . March 2020. doi:10.1007/s00417-020-04658-z 
7. Bertrand AL, Garcia JBS, Viera EB, Santos AM, Bertrand RH. Pupillometry: The Influence of Gender and Anxiety on the Pain Response.Pain Physician .:10.

8. Jain M, Devan S, Jaisankar D, Swaminathan G, Pardhan S, Raman R. Pupillary Abnormalities with Varying Severity of Diabetic Retinopathy.Sci Rep . 2018;8(1):5636. doi:10.1038/s41598-018-24015-9

9. Uwaezuoke SN, Ayuk AC, Ndu IK, Eneh C, Mbanefo NR, Ezenwosu OU. Vaso-occlusive crisis in sickle cell disease: current paradigm on pain management. JPR . 2018; Volume 11:3141-3150. doi:10.2147/JPR.S185582

10. Connelly MA, Brown JT, Kearns GL, Anderson RA, St Peter SD, Neville KA. Pupillometry: a non-invasive technique for pain assessment in paediatric patients. Archives of Disease in Childhood . 2014;99(12):1125-1131. doi:10.1136/archdischild-2014-306286

11. Sabourdin N, Diarra C, Wolk R, Piat V, Louvet N, Constant I. Pupillary Pain Index Changes After a Standardized Bolus of Alfentanil Under Sevoflurane Anesthesia: First Evaluation of a New Pupillometric Index to Assess the Level of Analgesia During General Anesthesia.Anesthesia ES Analgesia . 2019;128(3):467474. doi:10.1213/ANE.0000000000003681

12. Rollins MD, Feiner JR, Lee JM, Shah S, Larson M. Pupillary Effects of High-dose Opioid Quantified with Infrared Pupillometry:Anesthesiology . 2014;121(5):1037-1044. doi:10.1097/ALN.0000000000000384

Table 1 Means and Standard Deviations (SD) for Pupillometry Data

Overall $(\mathrm{n}=82)$ Males $(\mathrm{n}=44)$ Females $(\mathrm{n}=38)$ p-value

Minimum pupil size (mm) 3.52 (0.61) 3.57 (0.66) 3.47 (0.54) 0.495

Maximum pupil size (mm) 5.08 (0.78) 5.11 (0.85) 5.04 (0.71) 0.661

Maximum pupil constriction velocity (mm/s) -4.48 (1.05) -4.40 (0.90) -4.57 (1.21) 0.482

Average pupil constriction velocity (mm/s) -3.31 (0.73) -3.34 (0.76) -3.26 (0.69) 0.618

Dilation velocity $(\mathrm{mm} / \mathrm{s}) 1.03(0.31) 1.07(0.33) 0.99(0.27) 0.203$

Table 2 Mean Minimum and Maximum Pupil Sizes

Minimum Maximum

Age (years) \# participants pupil size pupil size

[mm] (SD) [mm] (SD)

$2-343.03(0.26) 4.83(0.29)$

$4-6193.25(0.41) 4.83(0.64)$

$7-9153.89(0.55) 5.49(0.64)$

10 - 12223.49 (0.67) $4.95(0.86)$

13 - $15123.84(0.65) 5.51$ (0.90)

$16-17103.40(0.50) 4.80(0.73)$

$\mathrm{SD}=$ standard deviation

Figure 1 Neuroptics Pupillometer

Figure 2 Scatter plot and regression line of pupil size and dilation velocity 


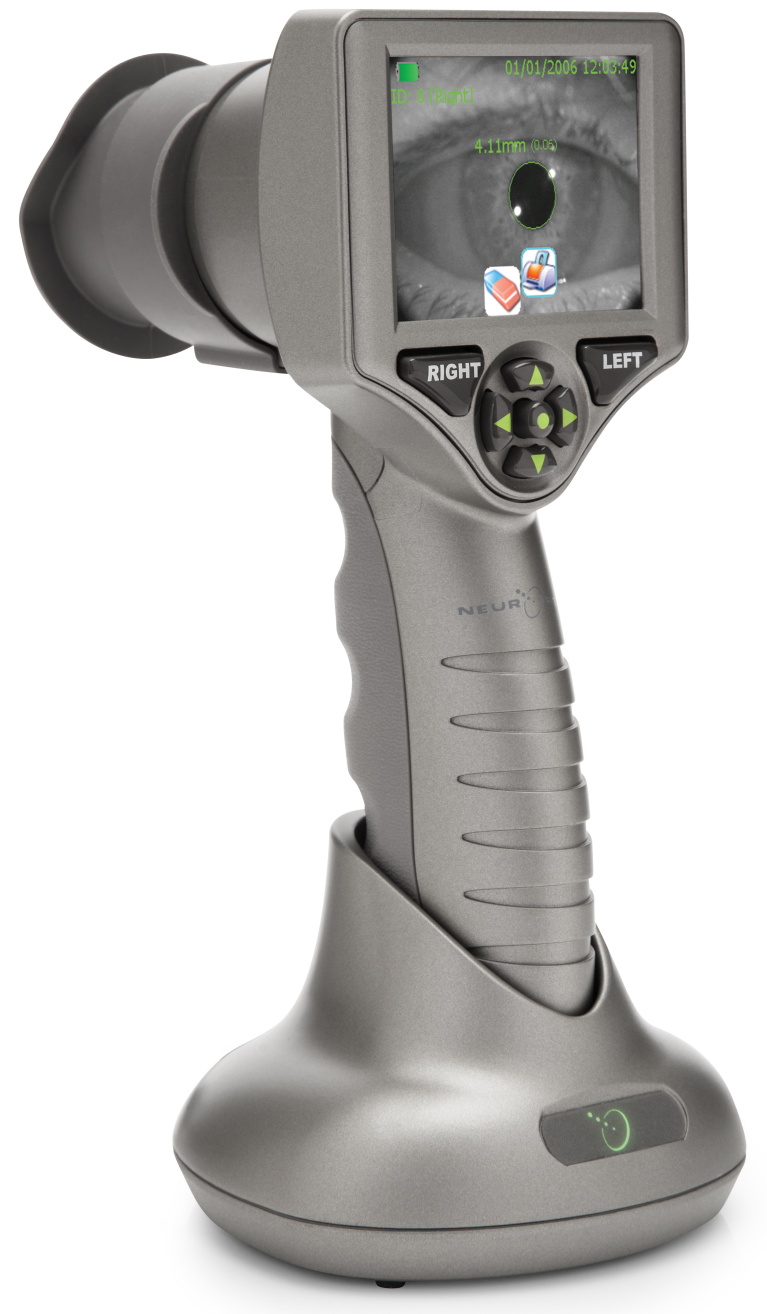



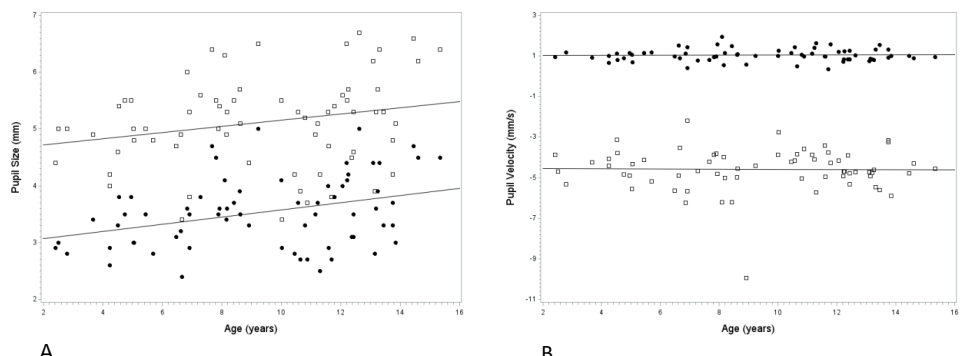

A.

B. 